\title{
Resonance Raman Characterization of the Forms of Ground-State 8-Substituted 7- Hydroxyquinoline Caged Acetate Compounds in Aqueous Solutions
}

\author{
Wen Li ${ }^{\mathrm{a}}$, Hui-Ying $\mathrm{An}^{\mathrm{a}}$, Chensheng Ma ${ }^{\mathrm{a}}$, Kyle T. Harris ${ }^{\mathrm{b}}$, \\ Timothy M. Dore ${ }^{b}$ and David Lee Phillips ${ }^{a}$ \\ ${ }^{a}$ Department of Chemistry, The University of Hong Kong, Hong Kong S.A.R., China \\ ${ }^{b}$ Department of Chemistry, University of Georgia, Athens, Georgia 30602-2556, USA
}

8-bromo-7-hydroxyquinolinyl group (BHQ) is a promising class of phototriggers with high enough sensitivity to be effective for both one-photon excitation (1PE) and two-photon excitation (2PE) applications in physiological environments. ${ }^{1}$ Similar to BHQ, 8-substituted 7-hydroxyquinolines, like 8-chloro-7-hydroxyquinoline (CHQ) and 8-cyano-7-hydroxyquinoline (CyHQ), are able to be useful for 1PE and 2PE and their acetate acids $\mathrm{CHQ}-\mathrm{OAc}$ and $\mathrm{CyHQ}-\mathrm{OAc}$ were also able to undergo photolysis reactions in neutral aqueous buffer solutions. ${ }^{2}$ To examine the substituent effect on the relative populations of the forms of the ground state species of 8 -substituted 7hydroxyquinolines, ultraviolet absorption and resonance Raman spectroscopy experiments were done for $\mathrm{CHQ}-\mathrm{OAc}$ and $\mathrm{CyHQ}-\mathrm{OAc}$ in acetonitrile $(\mathrm{MeCN})$, in $\mathrm{NaOH}-\mathrm{H}_{2} \mathrm{O} / \mathrm{MeCN}(60: 40, \mathrm{v} / \mathrm{v}, \mathrm{pH} 11 \sim 12)$, and in $\mathrm{H}_{2} \mathrm{O} / \mathrm{MeCN}(60: 40, \mathrm{v} / \mathrm{v}, \mathrm{pH}$ 6 7) solutions. These spectroscopic results were compared to those previously determined for the BHQ-OAc compound.

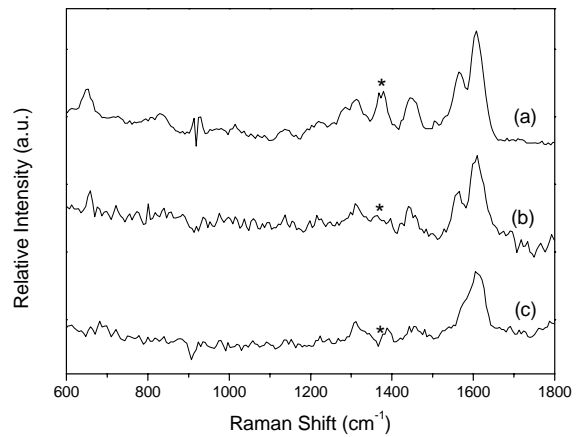

FIGURE 1. 266-nm resonance Raman spectra are shown for (a) BHQ-OAc, (b) CHQ-OAc and (c) $\mathrm{CyHQ}-\mathrm{OAc}$ obtained in $\mathrm{MeCN}$.

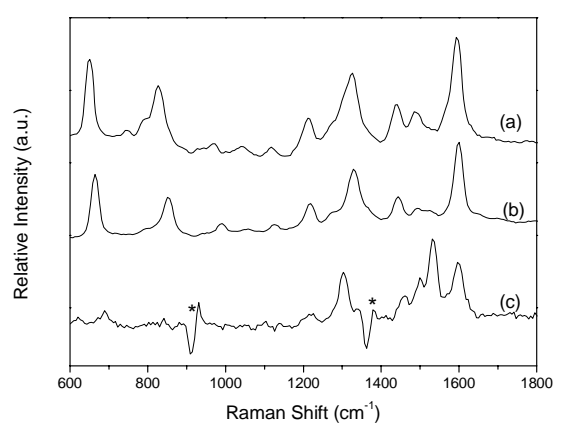

FIGURE 2. The 266-nm resonance Raman spectra are shown for (a) BHQ-OAc, (b) CHQ$\mathrm{OAc}$ and (c) CyHQ-OAc obtained in a NaOH$\mathrm{H} 2 \mathrm{O} / \mathrm{MeCN}(60: 40, \mathrm{v} / \mathrm{v}, \mathrm{pH}$ 11 12) solvent.

Figure 1 presents an overview of the resonance Raman spectra of the singlet ground states of the neutral forms of BHQ-OAc, CHQ-OAc and CyHQ-OAc. It is clear that 
the spectra of the neutral forms of CHQ-OAc and BHQ-OAc are very similar to each other and the spectrum of the neutral form of CyHQ-OAc appears different from the other two. Figure 2 also shows the anionic form of CyHQ-OAc is different from that of BHQ-OAc. These results suggest that the resonance Raman bands are noticeably affected by the electron-withdrawing nature of the substituents group.
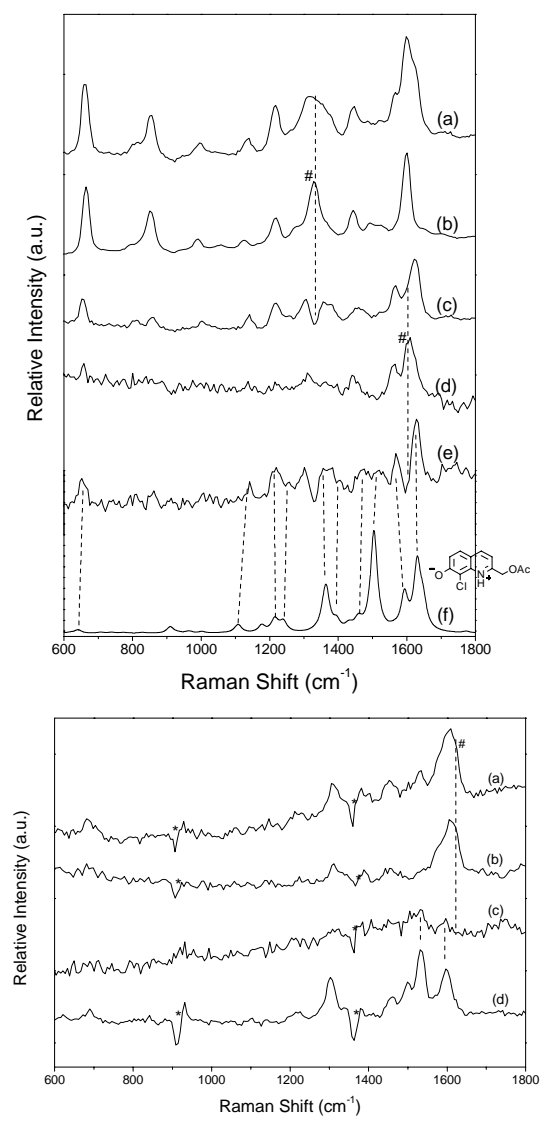

FIGURE 3. (a) The 266-nm resonance Raman spectrum of CHQ-OAc acquired in a mixed $\mathrm{H}_{2} \mathrm{O} / \mathrm{MeCN}$ solvent $(60: 40, \mathrm{v} / \mathrm{v}, \mathrm{pH}$ 6 7) is displayed. (b) The 266-nm resonance Raman spectrum of CHQ-OAc acquired in a mixed $\mathrm{NaOH}-\mathrm{H}_{2} \mathrm{O} / \mathrm{MeCN}$ solvent $(60: 40, \mathrm{v} / \mathrm{v}, \mathrm{pH}$ 11 12) is shown. (c) The bands remaining after subtraction of an appropriately scaled spectrum (b) from spectrum (a). (d) The 266-nm resonance Raman spectrum of CHQ-OAc acquired in $\mathrm{MeCN}$ is shown. (e) The bands remaining after subtraction of an appropriately scaled spectrum (d) from spectrum (c). (f) DFT calculated normal Raman spectrum of the singlet ground state of the tautomeric form of CHQ-OAc (whose structure is shown to the left of the spectrum) is displayed.

FIGURE 4. (a) The 266-nm resonance Raman spectrum of CyHQ-OAc acquired in a mixed $\mathrm{H}_{2} \mathrm{O} / \mathrm{MeCN}$ solvent $(60: 40, \mathrm{v} / \mathrm{v}, \mathrm{pH} 6 \sim 7)$ is shown. (b) The 266-nm resonance Raman spectrum of CyHQ-OAc acquired in a $\mathrm{MeCN}$ solvent is displayed. (c) The bands remaining after subtraction of an appropriately scaled spectrum (b) from spectrum (a) is shown. (d) The 266-nm resonance Raman spectrum of CyHQ$\mathrm{OAc}$ acquired in a mixed $\mathrm{NaOH}-\mathrm{H}_{2} \mathrm{O} / \mathrm{MeCN}$ solvent $(60: 40, \mathrm{v} / \mathrm{v}, \mathrm{pH} 11 \sim 12)$ is displayed.

Figures 3 and figure 4 indicate that the relative population of the tautomeric species of CHQ-OAc grows larger, but the tautomeric species of CyHQ-OAc can not be readily dsicerned in water rich solutions. The results reported here support the suggestion that the generation of the tautomeric forms of 7-hydroxyquinoline and its derivatives may be affected by steric and/or electronic effects of the 8-substituented group in aqueous environments. ${ }^{3}$

\section{REFERENCES}

1. (a) Fedoryak, O. D.; Dore, T. M. Org. Lett. 4,3419-3412 (2002). (b) Zhu, Y.; Pavlos, C. M.; Toscano, J. P.; Dore, T. M. J. Am. Chem. Soc. 128, 4267-4276 (2006).

2. Davis, M. J.; Kragor, C. H.; Reddie, K. G.; Wilson, H. C.; Zhu, Y.; Dore, T. M. J. Org. Chem. 74, 1721-1729 (2009).

3. An, H. Y.; Ma, C. S.; Nganga, J. L.; Zhu, Y.; Dore, T. M.; Phillips, D. L. J. Phys. Chem. A 113, 2831-2837 (2009). 